\title{
STUDIES OF PROPELLER AERODYNAMICS FOR UAV TETHERED SYSTEM DEVELOPMENT
}

\author{
Daniel Brun and Kiran Bhaganagar \\ The University of Texas at San Antonio
}

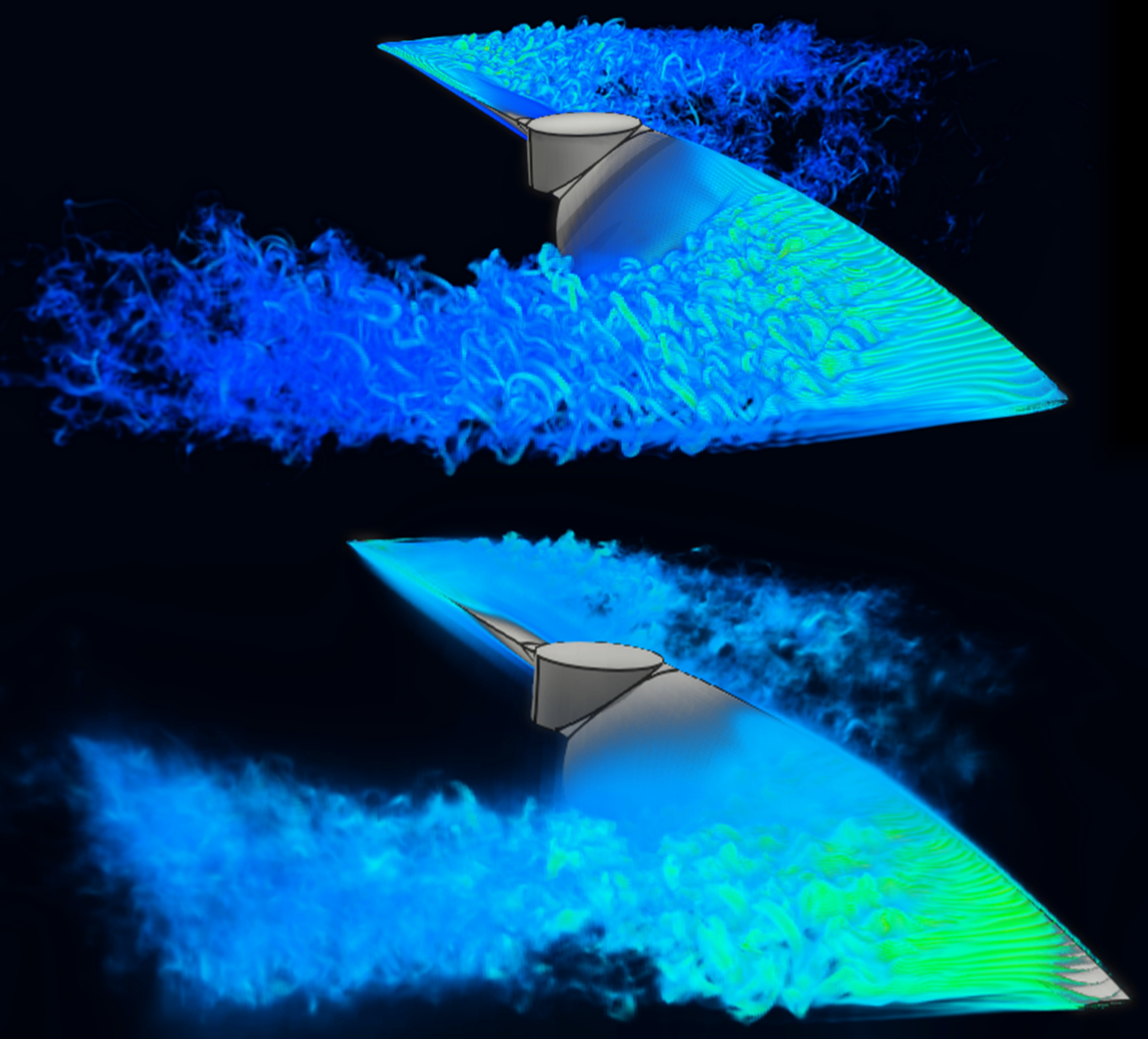

The placement of sensing systems mounted on multirotor UAV is essential to perform accurate measurements. In an effort to reduce the noise acquired by the sensors, product of the turbulence generated by the propeller of multirotor UAV, a tip vortex characterization is realized where quantitative and qualitative analysis is done to install the sensing systems on the quadcopter.

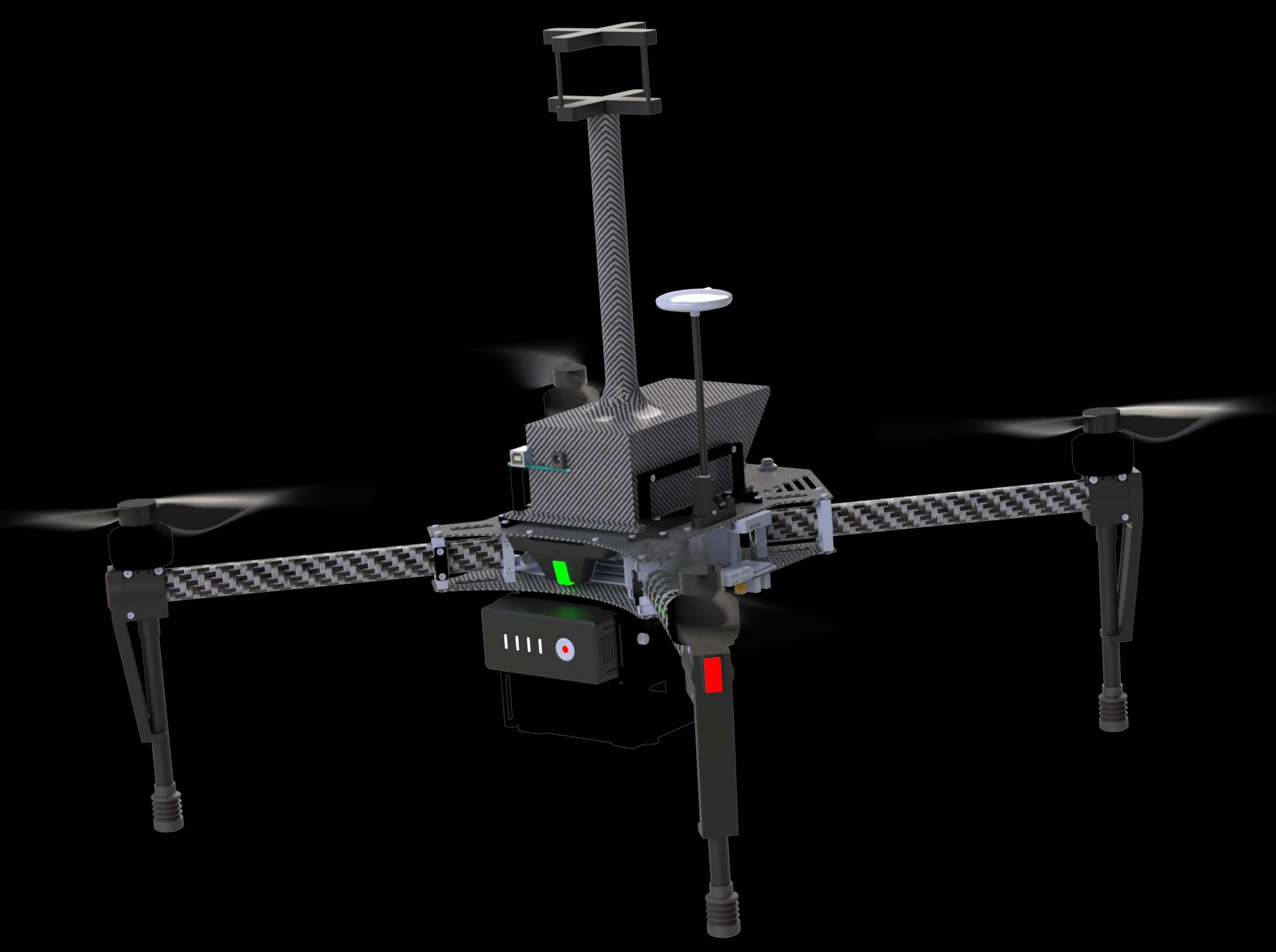

The introduction of a LiDAR in the system allows the collection of the physical environment. As example in the image, it can be observed Rowdy, our school mascot, and part of the Multidisciplinary Building on our Main Campus at UTSA.

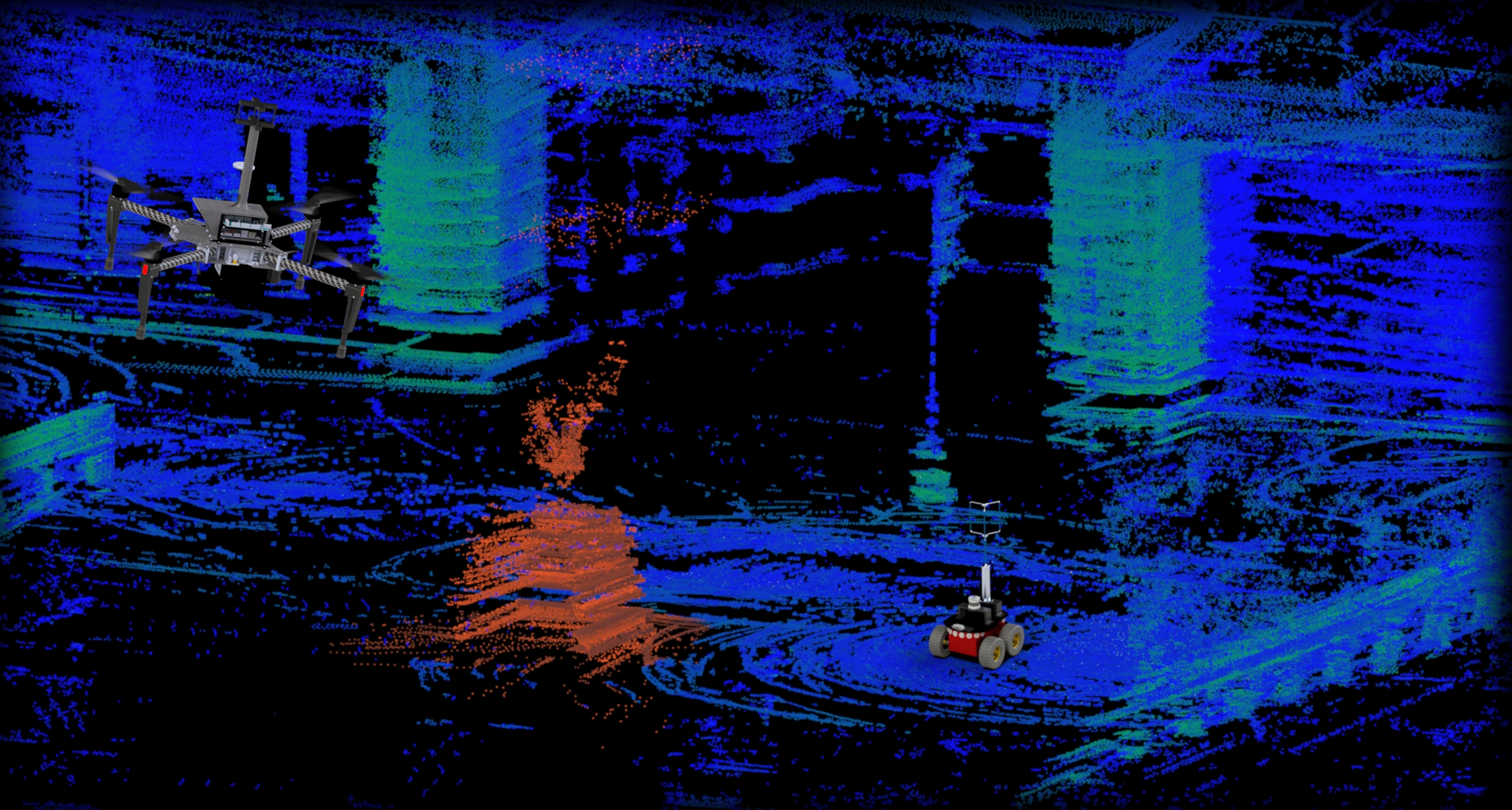

